\title{
Statistics of a noise-driven Manakov soliton
}

\author{
Stanislav A Derevyanko ${ }^{1,2}$, Jaroslaw E Prilepsky ${ }^{3}$, and Dennis \\ A Yakushev ${ }^{2}$ \\ ${ }^{1}$ Photonics Research Group, Aston University, Aston Triangle, B4 7ET, Birmingham, \\ United Kingdom \\ ${ }^{2}$ Institute for Radiophysics and Electronics National Academy of Sciences of Ukraine, \\ 12 Acad. Proscuri St., 61085, Khrakov, Ukraine \\ ${ }^{3}$ B.I. Verkin Institute for Low Temperature Physics and Engineering, National \\ Academy of Sciences of Ukraine, 47 Lenin Ave., 61103, Kharkov, Ukraine \\ E-mail: s.derevyanko@aston.ac.uk, astrayed@yandex.ru, den@ire.kharkov.ua
}

\begin{abstract}
We investigate the statistics of a vector Manakov soliton in the presence of additive Gaussian white noise. The adiabatic perturbation theory for Manakov soliton yields a stochastic Langevin system which we analyze via the corresponding FokkerPlanck equation for the probability density function (PDF) for the soliton parameters. We obtain marginal PDFs for the soliton frequency and amplitude as well as soliton amplitude and polarization angle. We also derive formulae for the variances of all soliton parameters and analyze their dependence on the initial values of polarization angle and phase.
\end{abstract}

PACS numbers: 42.81.Dp; 42.81.Gs

Submitted to: J. Phys. A: Math. Gen. 


\section{Introduction}

Nonlinear Shrodinger equation (NLSE) and its vector modifications have been studied in various contexts for more than 30 years. It has found applications in such areas as plasma physics, hydrodynamics, magnetism and nonlinear fibre optics. The NLSE, the so-called modified NLSE, and some of their vector counterparts (e.g. Manakov equations) are integrable and, as such, possess stable soliton solutions that exist due to the precise balance between the effects of nonlinearity and dispersion. Since solitons preserve their shape and internal properties during the propagation they have been widely used in fibre optics as information carriers [1, 2].

In most applications, however, integrable systems represent only the leading approximation of the real physical model. Quite often such models can be analysed using the concept of a nearly integrable system. In such systems the deviations from the initial integrable model are considered to be small and the underlying dynamics can be inferred via methods of perturbation theory [3. The important and distinct class of perturbations constitute random perturbations of different origin. In particular, in fibre optics, stochastic perturbations emulate the effects of the amplifier spontaneous emission as well as various fibre inhomogeneities [4, 5, 6]. The influence of random perturbations on solitons has been studied in different integrable systems including Korteveg-de-Vries (KdV) equation [7, 8, 9, NLSE [10, 11, 12, 13, 14] (see the latter Ref. for additional references), modified NLSE [15, 16, 17, and Manakov system [5, 6, 16]. Inasmuch as solitons in integrable systems are robust against small perturbations, the weak noise leads to random walk of soliton parameters (which is called jitter) as well as generation of dispersive radiation. In the sequel we will only be concerned with the additive white Gaussian noise (AWGN) model, that represents the simplest physically justified example of random perturbation. For such systems most celebrated result for soliton jitters is the so-called Gordon-Haus effect: the random walk of the soliton position due to the effect of weak AWGN [10]. The main tool used for studying the statistics of the soliton parameters for the aforementioned systems is the adiabatic soliton perturbation theory (see e.g. review paper [3] and references therein). Since the perturbation has stochastic nature the resulting system of equations for soliton parameters represents a Langevin system of stochastic equations with the multiplicative noise. For this Langevin system, in the vast majority of works on the subject the noise was assumed to be additive, and the equations were linearised. The assumption of the additive noise in the linearised Langevin equations can give rise only to the Gaussian probability dencity functions (PDF). However the exact statistics of the NLSE soliton, for example, in the presence of AWGN are not Gaussian as was shown in papers [11, 12, 13, 14. Gaussian approximation can only be used for approximate estimates of the integral characteristics of the PDF, like e.g. variances of soliton jitters.

In the present paper we will consider an integrable Manakov system driven by vector AWGN, a system which, in some respect, is a vector generalisation of the NLSE. Soliton of the Manakov equations possesses two additional parameters as compared to 
NLSE soliton. Doktorov and Kuten [16] demonstrated in the Gaussain approximation that timing jitter of the Manakov soliton can be managed by adjusting these two extra parameters. In the current paper we will show that not only timing jitter but all the remaining jitters can be managed in the similar fashion provided that the vector AWGN has anisotropic statistical properties. We will also obtain (for the first time to our knowledge) marginal PDFs for the parameters of the Manakov soliton, that describe correctly the non-Gaussian statistics of rare fluctuations. We will derive the exact statistics of the soliton parameters using the combination of the adiabatic soliton perturbation theory and the Fokker-Planck equation (FPE) approach, proceeding in the same way as in Refs. 13, 14] for the NLSE soliton.

The paper is organized as follows: in Section 2 we derive a system of Langevin equations with multiplicative noise using well established technique of the adiabatic perturbation theory for a single Manakov soliton. In section 3 the Fokker-Planck equation attached to the corresponding Langevin system is derived. In the same section we find the exact solution for the PDF of the amplitude-frequency jitter. Then we use a WKB approximation for the FPE to obtain a marginal PDF for the soliton amplitude and polarization angle. Eventually, in Section 4 the Gaussian limit is considered we calculate variances for all the soliton parameters. In Conclusion we summarise the results obtained.

\section{Stochastic adiabatic perturbation theory for Manakov soliton}

Consider a vector soliton propagating along a random medium, which is described by the perturbed Manakov equations

$$
\mathrm{i} \boldsymbol{\Psi}_{z}+\boldsymbol{\Psi}_{t t}+2\left(\boldsymbol{\Psi} \boldsymbol{\Psi}^{*}\right) \boldsymbol{\Psi}=\mathbf{n},
$$

where the components of vector $\boldsymbol{\Psi}=(u(z, t), v(z, t))^{T}$ represent two polarization states of a propagating vector field (e.g. electrical field in a birefringent optical fibre), whereas the r.h.s. of Eq.(11) accounts for the random noise (e.g. ASE) and its components have the following correlation properties:

$$
\begin{aligned}
& \left\langle n_{\alpha}(t, z)\right\rangle=\left\langle n_{\alpha}(t, z) n_{\beta}\left(t^{\prime}, z^{\prime}\right)\right\rangle=0, \\
& \left\langle n_{\alpha}(t, z) n_{\beta}^{*}\left(t^{\prime}, z^{\prime}\right)\right\rangle=D_{\alpha \beta} \delta\left(z-z^{\prime}\right) \delta\left(t-t^{\prime}\right), \quad \alpha, \beta=1,2 .
\end{aligned}
$$

Here matrix $\hat{D}$ is symmetric and positive definite.

In the absence of any perturbation the Manakov equations belong to the class of those which are integrable by the inverse scattering transform method. The general form of the one-soliton solution of Manakov equations $\boldsymbol{\Psi}_{0}$ is given by following expressions (see e.g. [6]):

$u(z, t)=2 \eta(z) \cos \beta(z) \operatorname{sech}\{2 \eta(z)[t-T(z)]\} \exp \{\mathrm{i} \alpha(z)-2 \mathrm{i}[t-T(z)] \xi(z)+\mathrm{i} \phi(z)\}$,

$v(z, t)=2 \eta(z) \sin \beta(z) \operatorname{sech}\{2 \eta(z)[t-T(z)]\} \exp \{\mathrm{i} \alpha(z)-2 \mathrm{i}[t-T(z)] \xi(z)-\mathrm{i} \phi(z)\}$,

and dependencies of the six soliton parameters on the propagation distance $z$ are:

$$
\eta(z)=\eta_{0}
$$


Statistics of a noise-driven Manakov soliton

$$
\begin{aligned}
& \xi(z)=\xi_{0}, \\
& \alpha(z)=4\left(\eta_{0}^{2}+\xi_{0}^{2}\right) z+\alpha_{0}, \\
& T(z)=-4 \xi_{0} z+T_{0}, \\
& \varphi(z)=\varphi_{0}, \\
& \beta(z)=\beta_{0},
\end{aligned}
$$

where the constants with zero subscripts are the values of soliton parameters at $z=0$.

Because the noise intensity is always small, the noise term, $\mathbf{n}(t, z)$, can be considered as a perturbation. In the presence of an arbitrary perturbation, $\mathbf{R}=\left(R_{1}, R_{2}\right)^{T}$, the soliton parameters become slowly varying functions of propagation distance $z$ and their evolution during propagation should be determined via the equations of the adiabatic soliton perturbation theory [6, 18, 19]:

$$
\begin{aligned}
\frac{\mathrm{d} \eta}{\mathrm{d} z} & =\operatorname{Re} \int \mathrm{d} t \mathbf{g}_{\eta}^{*}(t, z) \mathbf{R}(t, z), \\
\frac{\mathrm{d} \xi}{\mathrm{d} z} & =\operatorname{Re} \int \mathrm{d} t \mathbf{g}_{\xi}^{*}(t, z) \mathbf{R}(t, z), \\
\frac{\mathrm{d} \alpha}{\mathrm{d} z} & =4\left(\eta^{2}+\xi^{2}\right)+\operatorname{Re} \int \mathrm{d} t \mathbf{g}_{\alpha}^{*}(t, z) \mathbf{R}(t, z), \\
\frac{\mathrm{d} T}{\mathrm{~d} z} & =-4 \xi+\operatorname{Re} \int \mathrm{d} t \mathbf{g}_{T}^{*}(t, z) \mathbf{R}(t, z), \\
\frac{\mathrm{d} \varphi}{\mathrm{d} z} & =\operatorname{Re} \int \mathrm{d} t \mathbf{g}_{\varphi}^{*}(t, z) \mathbf{R}(t, z), \\
\frac{\mathrm{d} \beta}{\mathrm{d} z} & =\operatorname{Re} \int \mathrm{d} t \mathbf{g}_{\beta}^{*}(t, z) \mathbf{R}(t, z),
\end{aligned}
$$

where vector functions $\mathbf{g}_{i}$ can be expressed through derivatives of the single-soliton ansatz $\Psi_{0}$ with respect to appropriate soliton parameters. These functions can be written in the compact form as follows:

$$
\begin{aligned}
& \mathbf{g}_{\eta}=\frac{1}{2} \frac{\partial \boldsymbol{\Psi}_{0}}{\partial \alpha}, \quad \mathbf{g}_{\xi}=-\frac{\xi}{\eta} \mathbf{g}_{\eta}+\frac{1}{4 \eta} \frac{\partial \boldsymbol{\Psi}_{0}}{\partial T}, \\
& \mathbf{g}_{\varphi}=\frac{\csc (2 \beta)}{4 \eta} \frac{\partial \boldsymbol{\Psi}_{0}}{\partial \beta}, \quad \mathbf{g}_{\alpha}=-\cos (2 \beta) \mathbf{g}_{\varphi}+\frac{\xi}{2 \eta} \frac{\partial \boldsymbol{\Psi}_{0}}{\partial \xi}-\frac{1}{2} \frac{\partial \boldsymbol{\Psi}_{0}}{\partial \eta} \\
& \mathbf{g}_{T}=-\frac{1}{4 \eta} \frac{\partial \boldsymbol{\Psi}_{0}}{\partial \xi}, \quad \mathbf{g}_{\beta}=\frac{\cot (2 \beta)}{2 \eta} \mathbf{g}_{\eta}-\frac{\csc (2 \beta)}{4 \eta} \frac{\partial \boldsymbol{\Psi}_{0}}{\partial \varphi}
\end{aligned}
$$

The domain of integration in (17a)-(7f) should be from $-\infty$ to $\infty$. The set of equations (17a) $)$ - (7ff) with $\mathbf{R}(t, z)=\mathbf{n}(t, z)$ describes the fluctuations of soliton parameters under the action of AWGN provided that the interaction between the soliton and background radiation is negligible. The perturbation theory is not applicable in the limit $\eta \rightarrow 0$ and also in the limit when the polarization angle $\beta$ approaches values $\pi n / 2$ with an integer $n$. 


\section{Marginal PDFs for soliton parameters}

In this section we proceed to derive the FPE for all six soliton parameters and obtain the exact statistics of the amplitude-frequency jitter. We also use a WKB approach to derive marginal PDF for soliton amplitude and polarization angle. For simplicity we consider the case where the components of perturbation vector $\mathbf{n}$ are two independent noises with the same intensity $D$ :

$$
\left\langle n_{\alpha}(t, z) n_{\beta}^{*}\left(t^{\prime}, z^{\prime}\right)\right\rangle=D \delta_{\alpha \beta} \delta\left(z-z^{\prime}\right) \delta\left(t-t^{\prime}\right), \quad \alpha, \beta=1,2 .
$$

In that case the FPE reads as (see Appendix):

$$
\begin{aligned}
\frac{\partial P}{\partial z}= & \frac{\partial^{2}}{\partial \eta^{2}}\left[\frac{D \eta}{4} P\right]+\frac{\partial^{2}}{\partial \xi^{2}}\left[\frac{D \eta}{12} P\right]+\frac{\partial^{2}}{\partial T^{2}}\left[\frac{D \pi^{2}}{192 \eta^{3}} P\right]+\frac{\partial^{2}}{\partial \varphi^{2}}\left[\frac{D \csc (2 \beta)^{2}}{16 \eta} P\right]+ \\
& \frac{\partial^{2}}{\partial \beta^{2}}\left[\frac{D}{16 \eta} P\right]+\frac{\partial^{2}}{\partial \alpha^{2}}\left[\frac{D}{4}\left(\frac{1}{3 \eta}+\frac{\pi^{2}\left(\eta^{2}+3 \xi^{2}\right)}{36 \eta^{3}}+\frac{\cot ^{2}(2 \beta)}{4 \eta}\right) P\right]- \\
& \frac{\partial^{2}}{\partial \varphi \partial \alpha}\left[\frac{D \cot (2 \beta) \csc (2 \beta)}{16 \eta} P\right]-\frac{\partial^{2}}{\partial T \partial \alpha}\left[\frac{D \pi^{2} \xi}{96 \eta^{3}} P\right]-\frac{\partial}{\partial \eta}\left[\frac{3 D}{4} P\right]+\frac{\partial}{\partial T}[4 \xi P]- \\
& \frac{\partial}{\partial \beta}\left[\frac{D \cot (2 \beta)}{8 \eta} P\right]-\frac{\partial}{\partial \alpha}\left[4\left(\eta^{2}+\xi^{2}\right) P\right] .
\end{aligned}
$$

The boundary conditions in angular variables $\alpha, \varphi$ and $\beta$ are those of periodicity. In $\xi$,

$\eta$ and $T$ the PDF must decrease rapidly enough to provide normalization. At $\eta=0$ we assume that the $\eta$ component of the probability density current vanishes (see below) which means that no solitons are created of annihilated.

\subsection{Marginal PDF for amplitude-frequency jitter}

One can obtain in a closed form an autonomous FPE for the PDF of the amplitudefrequency jitter $P(\eta, \xi \mid z)$. Thin can be done by integrating out the redundant degrees of freedom in (9) by virtue of boundary conditions. Then the FPE for the PDF of the amplitude-frequency jitter is

$$
\frac{\partial P}{\partial z}=-\frac{\partial}{\partial \eta}\left[\frac{3 D}{4} P\right]+\frac{\partial^{2}}{\partial \eta^{2}}\left[\frac{D \eta}{4} P\right]+\frac{\partial^{2}}{\partial \xi^{2}}\left[\frac{D \eta}{12} P\right] .
$$

Since, without loss of generality, the initial value of $\xi$ may be put equal to zero the latter equation must be solved under the initial condition

$$
P(\eta, \xi \mid 0)=\delta(\xi) \delta\left(\eta-\eta_{0}\right)
$$

The $\eta$-component of the probability current vector is zero in the plane $\eta=0$ :

$$
\left.\left(\frac{3 D}{4} P-\frac{\partial}{\partial \eta}\left[\frac{D \eta}{4} P\right]\right)\right|_{\eta=0}=0 .
$$

The solution of this equation can be obtained by virtue of Fourier transform with respect to $\xi$ and Laplace transform with respect to $\eta$. The appearing equation is then solved 
Statistics of a noise-driven Manakov soliton

by the method of characteristics. Omitting the details, the solution is found to be

$$
P(\eta, \xi \mid z)=\frac{1}{2 \pi} \int_{-\infty}^{\infty} P_{k}\left(\eta \mid z^{\prime}\right) e^{-\mathrm{i} k \xi} \mathrm{d} k
$$

where the Fourier component is written as

$P_{k}\left(\eta \mid z^{\prime}\right)=\frac{\eta}{\eta_{0}} \frac{\alpha_{k}}{\sinh \left(\alpha_{k} z^{\prime}\right)} \exp \left[-\alpha_{k}\left(\eta+\eta_{0}\right) \operatorname{coth}\left(\alpha_{k} z^{\prime}\right)\right] \mathrm{I}_{2}\left(\frac{2 \alpha_{k} \sqrt{\eta \eta_{0}}}{\sinh \left(\alpha_{k} z^{\prime}\right)}\right)$.

Here $\mathrm{I}_{2}(\ldots)$ is the second-order modified Bessel function, $\alpha_{k}=k / \sqrt{3}$ and $z^{\prime}=D z / 4$. Integrating Eq.(13) over $\xi$ we obtain the marginal $\operatorname{PDF} P(\eta \mid z)$ :

$$
P(\eta \mid z)=\frac{1}{z^{\prime}} \frac{\eta}{\eta_{0}} \exp \left\{-\frac{\eta+\eta_{0}}{z^{\prime}}\right\} \mathrm{I}_{2}\left(\frac{2 \sqrt{\eta \eta_{0}}}{z^{\prime}}\right) .
$$

It is easy to find that the asymptotic of the PDF for the amplitude jitter is

$$
P(\eta \mid z) \approx \frac{\eta^{3 / 4}}{2 \sqrt{\pi z^{\prime}} \eta_{0}^{5 / 4}} \exp \left\{-\frac{\left(\sqrt{\eta}-\sqrt{\eta_{0}}\right)^{2}}{z^{\prime}}\right\}, \quad \eta \rightarrow \infty .
$$

The higher-order momenta of amplitude and frequency are:

$$
\begin{gathered}
\left\langle\eta^{n}\right\rangle=z^{\prime n} n ! \mathrm{L}_{n}^{2}\left(-\eta_{0} / z^{\prime}\right), \\
\left\langle\xi^{2 n}\right\rangle=\left.(-1)^{n} \frac{\partial^{2 n}}{\partial k^{2 n}}\left\{\operatorname{sech}^{3}\left(\alpha_{k} z^{\prime}\right) \exp \left[-\alpha_{k} \eta_{0} \tanh \left(\alpha_{k} z^{\prime}\right)\right]\right\}\right|_{k=0}, \quad n=1,2 \text {, etc. }
\end{gathered}
$$

The odd momenta of frequency vanish. In equation (17) for the amplitude momenta $\mathrm{L}_{n}^{2}(\ldots)$ are the generalized Laguerre functions. In particular, one can obtain the growth of the average amplitude with the propagation distance:

$$
\langle\eta\rangle=\eta_{0}+\frac{3 D}{4} z
$$

\subsection{Marginal PDF for polarization angle and amplitude. WKB-solution}

Marginal $\operatorname{PDF} P(\eta, \beta \mid z)$ satisfies the following FPE:

$\frac{\partial P}{\partial z}=-\frac{\partial}{\partial \eta}\left[\frac{3 D}{4} P\right]-\frac{\partial}{\partial \beta}\left[\frac{D \cot (2 \beta)}{8 \eta} P\right]+\frac{\partial^{2}}{\partial \eta^{2}}\left[\frac{D \eta}{4} P\right]+\frac{\partial^{2}}{\partial \beta^{2}}\left[\frac{D}{16 \eta} P\right]$.

We will use the WKB method to obtain a solution of the above equation [14, 20]. Assuming that noise amplitude, $D$, is small, we seek the solution in the form

$$
P=\exp [-S / D], \quad S=W+D W_{1}+D^{2} W_{2}+\ldots
$$

In the leading order in $D$ one obtains a Hamiltonian-Jacobi equation for the function $W$ :

$$
\frac{\partial W}{\partial z}+\frac{\eta}{4}\left[\frac{\partial W}{\partial \eta}\right]^{2}+\frac{1}{16 \eta}\left[\frac{\partial W}{\partial \beta}\right]^{2}=0 .
$$

The attached Freidlin-Wentzell Hamiltonian [20] is

$$
H=\frac{\eta}{4} p_{\eta}^{2}+\frac{1}{16 \eta} p_{\beta}^{2}
$$


where $p_{\eta}$ and $p_{\beta}$ represent the "momenta" canonically conjugated to the state variables, $\eta$ and $\beta$ correspondingly:

$$
p_{\eta}=\frac{\partial W}{\partial \eta}, \quad p_{\beta}=\frac{\partial W}{\partial \beta} .
$$

The solution of (22) is given by

$$
W=\int_{0}^{z}\left[p_{\eta}\left(z^{\prime}\right) \frac{\mathrm{d} \eta\left(z^{\prime}\right)}{\mathrm{d} z^{\prime}}+p_{\beta}\left(z^{\prime}\right) \frac{\mathrm{d} \beta\left(z^{\prime}\right)}{\mathrm{d} z^{\prime}}\right] \mathrm{d} z^{\prime}-E z,
$$

where $E=H$ is an integral of motion (see Refs. [14, 20] for details). The Hamiltonian trajectories in the integrand of (25) are found from Hamilton's equations:

$$
\begin{aligned}
& \frac{\mathrm{d} \eta}{\mathrm{d} z}=\frac{\eta}{2} p_{\eta}, \\
& \frac{\mathrm{d} \beta}{\mathrm{d} z}=\frac{1}{8 \eta} p_{\beta}, \\
& \frac{\mathrm{d} p_{\eta}}{\mathrm{d} z}=-\frac{1}{4} p_{\eta}^{2}+\frac{1}{16 \eta^{2}} p_{\beta}^{2}, \\
& \frac{\mathrm{d} p_{\beta}}{\mathrm{d} z}=0 .
\end{aligned}
$$

These trajectories are subject to the boundary conditions:

$$
\eta(0)=\eta_{0}, \quad \eta(z)=\eta, \quad \beta(0)=\beta_{0}, \quad \beta(z)=\beta .
$$

The solutions for the amplitude $\eta(z)$ and the polarization angle $\beta(z)$ are given by:

$$
\begin{gathered}
\eta(z)=\frac{z^{2} p_{\beta 0}^{2}+4 \eta_{0}^{2}\left(4+z p_{\eta 0}\right)^{2}}{64 \eta_{0}}, \\
\beta(z)=\beta_{0}-\arctan \left(\frac{2 \eta_{0} p_{\eta 0}}{p_{\beta 0}}\right)+\arctan \left(\frac{16 \eta_{0}^{2} p_{\eta 0}+z\left(p_{\beta 0}^{2}+4 \eta_{0}^{2} p_{\eta 0}^{2}\right)}{8 \eta_{0} p_{\beta 0}}\right) .
\end{gathered}
$$

One can now find constants $p_{\beta 0}$ and $p_{\eta 0}$ :

$$
\begin{aligned}
& p_{\beta 0}= \pm \frac{8 \sqrt{\eta} \sqrt{\eta_{0}} \tan \left(\beta-\beta_{0}\right)\left|\cos \left(\beta-\beta_{0}\right)\right|}{z}, \\
& p_{\eta 0}=\frac{4\left(-\sqrt{\eta_{0}} \pm \sqrt{\eta}\left|\cos \left(\beta-\beta_{0}\right)\right|\right)}{z \sqrt{\eta_{0}}} .
\end{aligned}
$$

From (25)-(26d) it follows that $W=E z$ so that we have two solutions of HamiltonJacobi equation (22):

$$
W_{ \pm}=\frac{4\left(\eta+\eta_{0} \pm 2 \sqrt{\eta} \sqrt{\eta_{0}}\left|\cos \left(\beta-\beta_{0}\right)\right|\right)}{z}
$$


The WKB approximation for the solution of (20) is written as a sum $P(\eta, \beta \mid z)=$ $P_{+}(\eta, \beta \mid z)+P_{-}(\eta, \beta \mid z)$, where $P_{ \pm}(\eta, \beta \mid z)$ is given by

$$
\begin{aligned}
P_{ \pm}= & C\left|\operatorname{det}\left(-\frac{\partial^{2} W_{ \pm}}{\partial q \partial q_{0}}\right)\right|^{1 / 2} \exp \left[\int_{0}^{z} B p \mathrm{~d} z^{\prime}\right] \times \\
& \exp \left[-\frac{1}{2} \int_{0}^{z} \operatorname{Tr} \frac{\partial^{2} H}{\partial q \partial p} \mathrm{~d} z^{\prime}\right] \exp \left[-\frac{W_{ \pm}}{D}\right] .
\end{aligned}
$$

Here for the sake of compactness we introduced the following $2 \mathrm{D}$ vectors: $q=(\eta, \beta)$, $q_{0}=\left(\eta_{0}, \beta_{0}\right), p=\left(p_{\eta}, p_{\beta}\right)$. Vector $B$ represents the part of the advection vector which is proportional to $D$ (with factor $D$ omitted). In our case $B=\{3 / 4, \cot (2 \beta) / 8 \eta\}$. The integrals in the exponential are taken along the corresponding Hamiltonian trajectories (28), (29), with the corresponding choice of sign in $p_{\eta 0}, p_{\beta 0}$. Factor $C$ is added to provide the normalization of the $\operatorname{PDF} P(\eta, \beta \mid z)$. Each multiplier in (31) can be evaluated separately for both trajectories:

$$
\begin{aligned}
& \operatorname{det}\left(-\frac{\partial^{2} W_{ \pm}}{\partial q \partial q_{0}}\right)=\frac{16}{z^{2}} \\
& -\frac{1}{2} \int_{0}^{z} \operatorname{Tr} \frac{\partial^{2} H}{\partial q \partial p} \mathrm{~d} z^{\prime}=-\frac{1}{4} \int_{0}^{z} p_{\eta} \mathrm{d} z^{\prime}=-\frac{1}{2} \ln \left(\frac{\eta}{\eta_{0}}\right),
\end{aligned}
$$

and

$$
\begin{aligned}
\int_{0}^{z} B p \mathrm{~d} z^{\prime} & =\int_{0}^{z}\left(\frac{3}{4} p_{\eta}+\frac{p_{\beta}}{8 \eta} \cot (2 \beta)\right) \mathrm{d} z^{\prime}=\int_{0}^{z}\left(\frac{3}{4} \frac{2 \eta^{\prime}}{\eta}+\cot (2 \beta) \beta^{\prime}\right) \mathrm{d} z^{\prime} \\
& =\frac{3}{2} \ln \left(\frac{\eta}{\eta_{0}}\right)+\frac{1}{2} \ln \left|\frac{\sin (2 \beta)}{\sin \left(2 \beta_{0}\right)}\right|
\end{aligned}
$$

This yields

$P_{ \pm}(\eta, \beta \mid z)=\frac{4 C}{z} \frac{\eta}{\eta_{0}} \sqrt{\frac{|\sin (2 \beta)|}{\left|\sin \left(2 \beta_{0}\right)\right|}} \exp \left[-\frac{4}{D z}\left(\eta+\eta_{0} \pm 2 \sqrt{\eta \eta_{0}}\left|\cos \left(\beta-\beta_{0}\right)\right|\right)\right]$.

To obtain the normalization constant $C$ we must integrate $P_{+}$and $P_{-}$over $\eta$ and $\beta$ and insure that the sum of the two integrals evaluates to unity. We perform the integration over $\beta$ first. Since in the WKB approximation the noise intensity, $D$, is assumed to be small, the main contribution into the integral over $\beta$ comes from the vicinity of the points where either $\cos \left(\beta-\beta_{0}\right)$ or $\sin \left(\beta-\beta_{0}\right)$ vanish. This implies $|\sin (2 \beta)| \approx\left|\sin \left(2 \beta_{0}\right)\right|$, and one obtains

$$
\int_{0}^{2 \pi} P(\eta, \beta \mid z) \mathrm{d} \beta \approx \frac{16 \pi C}{z} \frac{\eta}{\eta_{0}} \exp \left[-\frac{4}{D z}\left(\eta+\eta_{0}\right)\right] \mathrm{I}_{0}\left(\frac{8 \sqrt{\eta \eta_{0}}}{D z}\right),
$$

where we have used the identity $\int_{0}^{2 \pi} \cosh \{x|\cos t|\} d t=2 \pi \mathrm{I}_{0}(x)$. The expression above is nothing else but a WKB approximation for the marginal PDF for the amplitude, 
$P(\eta \mid z)$. Since the WKB approximation is valid under the assumption that $D \ll 1$ (or, more precisely, $D z \ll 1$ ) one can use the asymptotic of the Bessel function for the large values of its argument and observe an excellent agreement with exact formula (16) obtained earlier. Another integration over $\eta$ allows one to obtain the normalization constant $C$. The final result for the marginal $\operatorname{PDF} P(\eta, \beta \mid z)$ reads:

$P(\eta, \beta \mid z) \approx \frac{2}{\pi D z} \frac{\eta}{\eta_{0}} \sqrt{\frac{|\sin (2 \beta)|}{\left|\sin \left(2 \beta_{0}\right)\right|}} \exp \left[-\frac{4}{D z}\left(\eta+\eta_{0}\right)\right] \cosh \left[\frac{8 \sqrt{\eta \eta_{0}}}{D z}\left|\cos \left(\beta-\beta_{0}\right)\right|\right]$.

Note that this result is inapplicable for $\sin \left(2 \beta_{0}\right)=0$. This is a direct consequence of the fact that the adiabatic perturbation theory fails for such values of polarization angle (see discussion at the end of Section 2).

\section{Additive noise approximation}

Let us consider the case when the propagation distance $z$ is small. We assume that the deviations of soliton parameters from their initial values are small as well. Then for generic set of Langevin equations (A.1) we may linearize the advection vector around the initial position $q_{0}$ and assume that the perturbation functions $\mathbf{g}_{i}(q, t)$ depends on the initial value of vector $q_{0}$ rather than instantaneous value $q$ (as in Appendix we omit vector notations for $q$ and $f$ ). Under such assumptions Eq. (A.1) is transformed into a simple linear Langevin system with additive Gaussian noise:

$$
\frac{\mathrm{d} q_{i}(z)}{\mathrm{d} z}=f_{i}\left(q_{0}\right)+\left.\frac{\partial f_{i}}{\partial q}\right|_{q_{0}}\left(q-q_{0}\right)+s_{i}(z)
$$

with

$$
\begin{aligned}
& \left\langle s_{i}(z) s_{j}\left(z^{\prime}\right)\right\rangle=n_{i j} \delta\left(z-z^{\prime}\right), \\
& n_{i j}=2 G_{i j}\left(q_{0}\right)=\frac{1}{2} \operatorname{Re} \int_{-\infty}^{\infty} \mathbf{g}_{i}^{*} \hat{D} \mathbf{g}_{j} \mathrm{~d} t .
\end{aligned}
$$

(The elements of the matrix $\hat{G}$ are derived in Appendix, see (A.15)). The statistics of soliton parameters now become Gaussian. Similar approach is widely used to obtain approximate expressions for the variances of the soliton parameters in scalar noise-driven NLSE [4. Without loss of generality one can assume that $q_{0}$ corresponds to the steady state of the unperturbed system, i.e. $f_{i}\left(q_{0}\right)=0$. The mean and variances of vector $\delta q=q-q_{0}$ are now readily obtained in a tensor form:

$$
\begin{aligned}
& <\delta q(z)>=0, \\
& <\delta q(z) \otimes \delta q(z)>=2 \int_{0}^{z} \mathrm{~d} z^{\prime} \exp \left[\hat{A} z^{\prime}\right] \hat{G}\left(q_{0}\right) \exp \left[\hat{A}^{T} z^{\prime}\right] \\
& \hat{A}=\left.\frac{\partial f}{\partial q}\right|_{q_{0}} .
\end{aligned}
$$


Now assuming that $\Omega_{0}=T_{0}=0$, and redefining phase $\alpha \rightarrow \alpha-4 \eta_{0}^{2} z$ we obtain the following expressions for the soliton jitters:

$$
\begin{aligned}
& <\delta \eta^{2}>=\frac{z \eta_{0}}{2} \tilde{D}\left(\beta_{0}, \varphi_{0}\right) \\
& <\delta T^{2}>=\frac{z\left(3 \pi^{2}+256 z^{2} \eta_{0}^{4}\right)}{288 \eta_{0}^{3}} \tilde{D}\left(\beta_{0}, \varphi_{0}\right), \\
& <\delta \xi^{2}>=\frac{Z \eta_{0}}{6} \tilde{D}\left(\beta_{0}, \varphi_{0}\right) \\
& <\delta \varphi^{2}>=\frac{z}{8 \eta_{0} \sin ^{2}\left(2 \beta_{0}\right)} \tilde{D}\left(\pi / 2+\beta_{0}, \varphi_{0}\right), \\
& <\delta \beta^{2}>=\frac{1}{8 \eta_{0}} \tilde{D}\left(\pi / 2+\beta_{0}, \varphi_{0}\right)
\end{aligned}
$$

and

$$
\begin{aligned}
<\delta \alpha^{2}> & =2 F\left(\beta_{0}, \varphi_{0}\right) z+2 D_{12} \cot \left(2 \beta_{0}\right) \sin \left(2 \varphi_{0}\right) z^{2} \eta \\
& +\frac{128}{3} \tilde{D}\left(\beta_{0}, \varphi_{0}\right) \eta_{0} z^{3}\left(\eta_{0}+\frac{\xi^{2}}{12}\right)
\end{aligned}
$$

Here we have introduced the function

$$
\tilde{D}\left(\beta_{0}, \varphi_{0}\right)=D_{11} \cos ^{2} \beta_{0}+D_{22} \sin ^{2} \beta_{0}+D_{12} \cos \left(2 \varphi_{0}\right) \sin \left(2 \beta_{0}\right),
$$

and $D_{\alpha \beta}$ are the elements of the correlation matrix, see (3). Formula (41) for the timing jitter was first obtained by Doktorov and Kuten [16]. The expression for $F\left(\beta_{0}, \varphi_{0}\right)$ in (45) is rather involved:

$$
\begin{aligned}
& F\left(\beta_{0}, \varphi_{0}\right)=\frac{\pi^{2} \xi^{2}}{48 \eta_{0}^{3}} \tilde{D}\left(\beta_{0}, \varphi_{0}\right)+\frac{1}{576 \eta_{0}}\left\{D_{11}\left[129+4 \pi^{2}\right] \cos ^{2}\left(\beta_{0}\right)-36\left(2\left[D_{11}+D_{22}\right]+\right.\right. \\
& \left.D_{11} \cos \left(2 \beta_{0}\right)\right)+9 \cos \left(2 \beta_{0}\right)\left(D_{22}\left[11+\cot ^{2}\left(\beta_{0}\right)\right]+4 D_{12} \cos \left(2 \varphi_{0}\right) \cot \left(2 \beta_{0}\right)\right)+ \\
& 54 D_{11} \sin ^{2}\left(\beta_{0}\right)+4 D_{22}\left[48+\pi^{2}\right] \sin ^{2}\left(\beta_{0}\right)+9 D_{11} \tan ^{2}\left(\beta_{0}\right) \sin ^{2}\left(\beta_{0}\right)+ \\
& \left.4 D_{12}\left[12+\pi^{2}\right] \cos \left(2 \varphi_{0}\right) \sin \left(2 \beta_{0}\right)\right\}
\end{aligned}
$$

Eqs.(40)-(42) can be endowed with a simple interpretation. First one notices that a single soliton ansatz (4), (15) can be transformed into a single soliton anzatz of the scalar NLSE via a unitary transformation:

$$
\begin{aligned}
& \boldsymbol{\Psi}=\hat{T}(z) \tilde{\boldsymbol{\Psi}}, \quad \tilde{\boldsymbol{\Psi}}=\left(\begin{array}{c}
u_{0} \\
0
\end{array}\right), \\
& T(z)=\left[\begin{array}{cc}
\cos \beta(z) & -\sin \beta(z) \\
\sin \beta(z) & \cos \beta(z)
\end{array}\right]\left[\begin{array}{cc}
\mathrm{e}^{\mathrm{i} \varphi(z)} & 0 \\
0 & \mathrm{e}^{-\mathrm{i} \varphi(z)}
\end{array}\right],
\end{aligned}
$$

where $u_{0}=2 \eta \operatorname{sech}[2 \eta(t-T)] \exp [\mathrm{i} \alpha-2 \mathrm{i}(t-T) \xi]$ is a single soliton solution of the scalar NLSE. Since in the additive noise approximation we are interested only in small deviations of the soliton parameters from their initial positions we may assume $\hat{T}(z) \approx \hat{T}(0)$. We can then observe that applying transformation $\hat{T}^{-1}$ leaves the l.h.s. of perturbed Manakov equation (11) invariant. As for the noise in the r.h.s. it is transformed into $\tilde{\mathbf{n}}=\hat{T}^{-1} \mathbf{n}$, which is again AWGN with the correlation matrix $\hat{\tilde{D}}=\hat{T}^{-1} \hat{D} \hat{T}$. 
Therefore the variances of the amplitude, phase and frequency of Manakov soliton $\boldsymbol{\Psi}_{0}$ in the additive noise approximation are equal to those of the scalar NLSE soliton but with the normalized noise intensity: $D \rightarrow(\hat{\tilde{D}})_{11}=\tilde{D}\left(\beta_{0}, \varphi_{0}\right)$. The variances of soliton jitters for scalar NLSE in this approximation are known [4, 14. One can observe that formulae (40)-(42) coincide with those given in [4] for amplitude, position and soliton frequency of a NLSE soliton provided that the noise intensity is now phase and polarization dependent $D=\tilde{D}\left(\beta_{0}, \varphi_{0}\right)$.

This has a few interesting consequences. First we observe that for the isotropic noise when the correlation matrix $\hat{D}$ is proportional to the identity matrix, $\hat{D}=D \hat{I}$, amplitude, timing and frequency jitter do not depend on polarization or phase at all and we recover exactly the result for the scalar NLSE. In general case we must analyze the behavior of the function $\tilde{D}\left(\beta_{0}, \varphi_{0}\right)$. One can readily see that one of the local minima is given by:

$$
\varphi=\frac{\pi}{2}, \quad \beta=\frac{1}{2} \arctan \left[\frac{2 D_{12}}{D_{22}-D_{11}}\right] .
$$

The minimal value of the function $\tilde{D}\left(\beta_{0}, \varphi_{0}\right)$ is

$$
\tilde{D}_{\min }=\frac{D_{11}+D_{22}}{2}-\frac{1}{2} \sqrt{4 D_{12}^{2}+\left(D_{22}-D_{11}\right)^{2}} \text {. }
$$

Because of matrix $\hat{D}$ being positive definite $\operatorname{det} \hat{D} \geq 0$, and $\tilde{D}_{\min }$ is always non-negative, as it should be. If det $\hat{D}=0$, i.e. $D_{11} D_{22}=D_{12}^{2}$, then one can in principle dispose of the soliton jitters completely (in the additive noise approximation). In practice, one can make soliton jitters almost negligible, provided that the determinant of the correlation matrix $\hat{D}$ is close to zero.

\section{Conclusion}

To sum up, we have considered the non-Gaussian statistics of Manakov soliton and demonstrated the dependence of the variances of all soliton parameters on phase mismatch $\varphi_{0}$ and polarization angle $\beta_{0}$. We derived the FPE describing the statistics of all soliton parameters. Using this approach we were able to work out the analytical expressions for the marginal PDFs for soliton amplitude $\eta$, frequency $\xi$ and polarization angle $\beta$. The statistics of the soliton frequency and amplitude appeared very much alike for scalar and vector NLSE in the case when vector AWGN is isotropic. This should not be considered as a surprise since for the isotropic perturbation of the Manakov system there exists a unitary transformation of the Manakov soliton into a NLSE soliton (localized in a single polarization) which leaves equation (11) invariant [6]. The situation however changes when one considers cross-correlations between noises in different polarizations. In the presence of a noisy perturbation with cross-correlations the symmetry related to the unitary transformation of a vector NLSE (space rotation and phase transformation) is broken. This means that though the relation between Manakov and NLSE solitons still exists the statistics of the soliton components becomes 
polarization and phase dependent. Controlling the initial values of the polarization and the phase one can effectively manage the magnitude of each soliton jitter separately (in particular timing jitter). If the determinant of the noise correlation matrix is small one can effectively decrease the variance of a given soliton jitter and make it negligible. This is impossible for an isotropic noise and for a scalar NLSE. It means that the Manakov soliton is in general more robust against noisy perturbations than its scalar counterpart. The correlations between different polarization noise components can play a positive role in suppressing soliton jitters.

The results obtained in the current paper rely on a series of assumptions. Firstly, the use of the adiabatic perturbation theory implies that we neglect the feedback of the linear radiation on the soliton. This is true for relatively small distances, where soliton parameters do not experience drastic deviations over the very short scales. Secondly, the perturbation theory developed here is unapplicable when the field polarization is close to linear $(\sin (2 \beta) \approx 0)$. Such cases should be considered separately (see $[6]$ and references therein). And finally, we have used additive noise (or Gaussian) approximation to obtain the variances of the soliton jitters. This approximation works fairly well for relatively small distances $(D z \ll 1)$ and gives reliable estimates for the variances. One should not be thinking, however, that the statistics of the tails of the PDF are Gaussian. In fact they are not as we have shown in Section 3, The FPE approach developed here may come handy when studying the statistics of the rare events forming the tails of the soliton PDF.

\section{Acknowledgments}

We wish to thank Sergei Turitsyn for stimulating discussions.

\section{Appendix. Derivation of the Fokker-Planck equation}

The purpose of this Appendix is the derivation of the FPE which describes the $\mathrm{PDF}$ of the system with multi-component multiplicative noise. Suppose $q^{s}(z)=$ $\left(q_{1}^{s}(z), \ldots, q_{N}^{s}(z)\right)$ is a solution of set of $N$ stochastic equations with $M$-component multiplicative white complex gaussian noise

$$
\frac{\mathrm{d} q_{i}(z)}{\mathrm{d} z}=f_{i}(q)+\operatorname{Re} \int_{-\infty}^{\infty} \mathrm{d} t \mathbf{g}_{i}^{*}(q, t) \mathbf{n}(t, z),
$$

with

$$
\begin{aligned}
& \mathbf{n}(t, z)=\left(n_{1}(t, z), \ldots, n_{M}(t, z)\right) \\
& \mathbf{g}_{i}(q, t)=\left(g_{i, 1}(q, t), \ldots, g_{i, M}(q, t)\right) .
\end{aligned}
$$

and

$$
\begin{aligned}
& \left\langle n_{\alpha}(z, t)\right\rangle=\left\langle n_{\alpha}(z, t) n_{\beta}\left(z^{\prime}, t^{\prime}\right)\right\rangle=0, \\
& \left\langle n_{\alpha}(z, t) n_{\beta}^{*}\left(z^{\prime}, t^{\prime}\right)\right\rangle=D_{\alpha \beta} \delta\left(z-z^{\prime}\right) \delta\left(t-t^{\prime}\right) .
\end{aligned}
$$


Statistics of a noise-driven Manakov soliton

Here and in the sequel we will omit vector notations for the $N$-dimensional vectors $q$ and $f$ in order not to confuse them with vectors $\mathbf{g}_{i}$ and $\mathbf{n}$ since they have different dimensionality.

We are interested in equation for the PDF of the solution of (A.1), $P(q, z)$. This PDF can be expressed in the form of

$$
P(q, z)=\left\langle\delta\left(q-q^{s}(z)\right)\right\rangle .
$$

Differentiating the latter equation with respect to $z$ and substituting the derivatives $\mathrm{d} q_{i}^{s}(z) / \mathrm{d} z$ one obtains

$\frac{\partial P}{\partial z}=-\sum_{i} \frac{\partial}{\partial q_{i}}\left[f_{i} P\right]-\sum_{i} \operatorname{Re} \frac{\partial}{\partial q_{i}} \int_{-\infty}^{\infty} \mathrm{d} t\left\{\mathbf{g}_{i}^{*}(q, t)\left\langle\delta\left(q-q^{s}(z)\right) \mathbf{n}(t, z)\right\rangle\right\}$,

where the average in the latter term can be evaluated by virtue of Furutsu-Novikov formula [21] that in the case of vector white complex noise $\mathbf{n}(t, z)$ is generalized to

$$
\left\langle\Theta\left[\mathbf{n}, \mathbf{n}^{*}\right] \mathbf{n}(t, z)\right\rangle=\hat{D}\left\langle\frac{\delta \Theta\left[\mathbf{n}, \mathbf{n}^{*}\right]}{\delta \mathbf{n}^{*}(t, z)}\right\rangle,
$$

where $\Theta\left[\mathbf{n}, \mathbf{n}^{*}\right]$ is an arbitrary functional of noise and matrix $\hat{D}$ has elements $D_{\alpha \beta}$ (A.5). Since

$$
\begin{aligned}
& \frac{\delta n_{\alpha}^{*}(t, z)}{\delta n_{\beta}^{*}\left(t^{\prime}, z^{\prime}\right)}=\delta_{\alpha \beta} \delta\left(z-z^{\prime}\right) \delta\left(t-t^{\prime}\right), \\
& \frac{\delta n_{\alpha}(t, z)}{\delta n_{\beta}^{*}\left(t^{\prime}, z^{\prime}\right)}=0, \\
& \frac{\delta q_{k}^{s}(z)}{\delta \mathbf{n}^{*}(t, z)}=\frac{1}{2} \theta(0) \mathbf{g}_{k}\left(q^{s}(z), t\right), \quad \theta(0)=\frac{1}{2},
\end{aligned}
$$

(where in the latter formula we have used Eq.A.1) and assumed symmetric Stratonovich regularization of the white noise) we arrive at

$$
\left\langle\delta\left(q-q^{s}(z)\right) \mathbf{n}(t, z)\right\rangle=-\frac{1}{4} \sum_{k} \frac{\partial}{\partial q_{k}}\left[\hat{D} \mathbf{g}_{k}(q, t) P(\mathbf{q}, z)\right] .
$$

Therefore for the $\operatorname{PDF} P(q, z)$ we arrive at the FPE equation:

$$
\frac{\partial}{\partial z} P(q, z)=-\sum_{i} \frac{\partial}{\partial q_{i}}\left[G_{i}(q) P(q, z)\right]+\sum_{i, k} \frac{\partial^{2}}{\partial q_{i} \partial q_{k}}\left[G_{i k}(q) P(q, z)\right] .
$$

Here the components of the advection vector and the diffusion matrix $\left(G_{i}\right.$ and $G_{i j}$ respectively) are defined as

$$
\begin{aligned}
& G_{i}(q)=f_{i}(q)+\frac{1}{4} \operatorname{Re} \sum_{k} \int_{-\infty}^{\infty} \mathrm{d} t \frac{\partial \mathbf{g}_{i}^{*}}{\partial q_{k}} \hat{D} \mathbf{g}_{k}, \\
& G_{i k}(q)=\frac{1}{4} \operatorname{Re} \int_{-\infty}^{\infty} \mathrm{d} t \mathbf{g}_{i}^{*} \hat{D} \mathbf{g}_{k},
\end{aligned}
$$

where $i, k=1, \ldots N$. 


\section{References}

[1] Agraval G 2001 Nonlinear fiber optics, 3-rd ed (Academic Press, San Diego).

[2] Hasegawa A and Kodama Y 1995 Solitons in optical communications (Oxford University Press, New York).

[3] Kivshar Yu and Malomed B 1989 Rev. Mod. Phys. 61763.

[4] Iannone E, Matera F, Mecozzi A, and Settembre M 1998 Nonlinear Optical Communication Networks (Wiley, New York).

[5] Wai P K A and Menyuk C 1996 J. Lightwave Technol. 14148.

[6] Lakoba T and Kaup D 1997 Phys. Rev. E 566147.

[7] Scalerandi M, Romano A, and Condat C 1998 Phys. Rev. E 584166.

[8] de Bouard A, Debussche A, and Tsutsumi Y 1999 J. Funct. Anal. 169532.

[9] Garnier J 2001 J. Stat. Phys. 105789.

[10] Gordon J and Haus H 1986 Opt. Lett. 11665.

[11] Malomed B and Flytzanis N 1993 Phys. Rev. E 48 R5.

[12] Falkovich G, Kolokolov I, Lebedev V, Mezentsev V, and Turitsyn S 2004 Physica D 1951.

[13] Derevyanko S, Turitsyn S and Yakushev D 2003 Opt. Lett. 282097.

[14] Derevyanko S, Turitsyn S and Yakushev D 2005 J. Opt. Soc. Am. B 22743.

[15] Doktorov E and Kuten I 2001 Europhys. Lett. 5322.

[16] Doktorov E and Kuten I 2001 Nonlinear Phenomena in Complex Systems 41.

[17] Yakushev D 2005 Radiophysics and electronics 10294.

[18] Midrio M and Wabnitz S 1996 Phys. Rev. E 545743.

[19] Shchesnovich V and Doktorov E 1997 Phys. Rev. E 557626.

[20] Gaspard P 2002 J. Stat. Phys. 10657.

[21] Garcia-Ojavo J and Sancho J, Noise in spatially extended systems (Springer, Berlin, 1999). 